\title{
A New Method to Solve Numeric Solution of Nonlinear Dynamic System
}

\author{
Min Hu and Fengjun Li \\ School of Mathematics and Statistics, Ningxia University, Yinchuan 750021, China \\ Correspondence should be addressed to Fengjun Li; fjli@nxu.edu.cn
}

Received 25 July 2016; Accepted 17 October 2016

Academic Editor: Lihua Wang

Copyright ( $) 2016$ M. Hu and F. Li. This is an open access article distributed under the Creative Commons Attribution License, which permits unrestricted use, distribution, and reproduction in any medium, provided the original work is properly cited.

\begin{abstract}
It is well known that the cubic spline function has advantages of simple forms, good convergence, approximation, and second-order smoothness. A particular class of cubic spline function is constructed and an effective method to solve the numerical solution of nonlinear dynamic system is proposed based on the cubic spline function. Compared with existing methods, this method not only has high approximation precision, but also avoids the Runge phenomenon. The error analysis of several methods is given via two numeric examples, which turned out that the proposed method is a much more feasible tool applied to the engineering practice.
\end{abstract}

\section{Introduction}

With the advancement of science and technology, the nonlinear problems have appeared in many fields. The conventional linear approach cannot meet the requirements of solving nonlinear problems. Therefore, the nonlinear dynamic has been born, which aims at discovering complexity sciences and provides an innovative way to recognize the real and complicated systems. Bifurcation and chaos are the two typical complex dynamic behaviors of nonlinear dynamic systems. In a sense, nonlinear dynamic system is the key topic of nonlinear problems, which can study the chaotic or disordered issue and excavate the complex law of them $[1,2]$. For a nonlinear dynamic system, a suitable nonlinear mathematical model should be established to reflect the inherent law of the data and then obtain the characteristics of the system. However, the nonlinear dynamic is diverse, and it changes depending on the previous state in a more complex way. Consequently, there has encountered insurmountable obstruction for applying in the practical engineering. With the existence of complicated chaotic state, it is very difficult to obtain analytic solution in general case. Naturally, people give up solving the exact solution and concentrate on studying the method with characteristics of high approximation precision and easy operation in order to describe the unknown system state.
After years of accumulation and development, there are too many methods to solve the numerical solution of nonlinear dynamic systems; the main methods are as follows: perturbation method [3], averaging method [4], Runge-Kutta method [5], Euler method [6], gradient method [7] and so on. Regretfully, these methods have certain advantages in solving certain system but obtain unappealing outcomes when solving problems of general nonlinear dynamic systems, like the lower precision, the complicity and large calculation quantity, Runge phenomenon, and so forth. So now comes the question, can we find the effective method with high approximation precision as well as avoiding the Runge phenomenon to study the nonlinear dynamic systems? It is well known that the cubic spline function has advantages of good convergence, approximation, stability, and secondorder smoothness. And not only that, the cubic spline function does not exist Runge phenomenon due to the restrictions of interpolation conditions.

Given this, the purpose of this paper is to construct a new method to solve the problem mentioned above which replaces the integrand used in the existing way by the constructed cubic spline function. The paper is organized as follows. In Section 2, we recall the basic concepts from approximation theory, such as modulus of continuity. In addition, some basic knowledge of dynamic system and definition of cubic spline function are introduced. In the 
following, the corresponding boundary conditions of general cubic spline function are given and a particular class of cubic spline function is constructed. In Section 4, we prove our conclusions. Two numerical results are provided in Section 5 by using the previously obtained theoretical results, and the result indicates that the numerical approach based on the cubic spline function has faster convergence and higher approximation precision than existing methods. In addition, the cubic spline function can approximate the analytic solution of nonlinear dynamic system very well. In Section 6, we briefly summarize our conclusions and foresee problems for the further study.

\section{Preliminaries}

There is a fixed rule in the dynamic system to describe how the point changes over time in the space, and a continuous dynamic system is often represented as a set of differential equations [8]:

$$
\dot{x}=f(x),
$$

where $x: t \rightarrow x \in R^{N}$, a time variable function, is used to describe the state of system $f$; at least, it is a continuously differentiable function defined on Euclid space $R^{N}$ or a subspace $U \in R^{N}$.

The linear combination of linearly independent solutions $\left\{x^{1}(t), \ldots, x^{N}(t)\right\}$ can be used to express the general solution of the linear dynamic system. But it is unable to work out general solution of the nonlinear dynamic system. And for all we know, the initial value problem of nonlinear dynamic system is as follows [9]:

$$
\begin{aligned}
\dot{x} & =f(x, t), \\
x\left(t_{0}\right) & =x_{0},
\end{aligned}
$$

$$
x \in R^{N} \text {. }
$$

There is at least one solution from $t=0$ on the interval of $t \in(-c, c)$. However, it is impossible to give a general calculation rule like the linear dynamic system. Consequently, the numerical method is commonly used for studying the solution of nonlinear dynamic system.

This so-called numerical method is actually a dispersed method. We can obtain the approximate solutions $x_{1}, x_{2}, \ldots, x_{n}$ of unknown function values $x\left(t_{1}\right), x\left(t_{2}\right), \ldots$, $x\left(t_{n}\right)$ on a series of discrete points $t_{1}, t_{2}, \ldots, t_{n}$. The discrete points of independent variable can be fixed before and also can select different step length along with the different nodes. $t_{j}, j \in 0,1,2, \ldots, n$, are generally equidistant nodes, namely, $t_{1}=t_{0}+h, t_{2}=t_{0}+2 h, \ldots, t_{n}=t_{0}+n h$, where step length $h>0, x_{1}, x_{2}, \ldots, x_{n}$, are commonly referred to as numerical solution of initial value problem.

Considering the nonlinear dynamic system with initial value as form (2), we obtain

$$
x\left(t_{j+1}\right)-x\left(t_{j}\right)=\int_{t_{j}}^{t_{j+1}} f(x(t), t) \mathrm{d} t .
$$

Definition 1. For every $r \in N^{+}, \delta>0$, any function $f \in[a, b]$, the definition of $r$ th order modulus of smoothness of $f$ is as follows [10]:

$$
w_{r}(f, \delta):=\sup _{a \leq x, x+r t \leq b,|t| \leq \delta}\left|\Delta_{t}^{r} f(x)\right|
$$

where $\Delta_{t}^{r} f(x):=\Delta_{t}^{1} \Delta_{t}^{r-1} f(x)$ and $\Delta_{t}^{1} f(x):=f(x+t)-f(x)$. When $r=1$, we have $w_{1}(f, \delta)=w(f, \delta)$. That is, the firstorder modulus of smoothness of $f$ is the same as modulus of continuity of $f$.

The smooth interpolating curves of spline function are unlikely to reveal the large oscillations feature of highdimension polynomials. It has been widely used in cartography, pyramidal, and numerical solution methods. For example, spline function may be used for solutions of initial value problems in ordinary differential equations [11, 12].

Let $\Delta:=\left\{a=t_{1}<t_{2}<\cdots<t_{n}=b\right\}$ be a partition of the interval $[a, b]$.

Definition 2. A cubic interpolation function $S$ defined on $\Delta$ is a real function $S:[a, b] \rightarrow R$ with the characteristics [13]:

(a) $S \in C^{2}[a, b]$; namely, $S$ is twice continuously differentiable on $[a, b]$.

(b) $S$ coincides with a polynomial of degree three on every subinterval $\left[t_{j}, t_{j+1}\right], j=1,2, \ldots, n$.

\section{Construct the Cubic Spline Function}

Cubic spline interpolation function has advantages of good stability, convergence, and high approximation accuracy, which has second-order smoothness at the interpolation nodes and avoids the Runge phenomenon on account of the limitation of the interpolation condition. So we hope to replace the integrand $f$ in (3) by the constructed cubic spline function $S(t)$. Meanwhile $S(t)$ satisfies the interpolation conditions, $y_{j}=f\left(x_{j}, t_{j}\right)(j=1,2, \ldots, n), S\left(t_{j}\right)=y_{j}$, $j=1,2, \ldots, n$, and $\left\{t_{j}\right\}$ are isometric interpolation nodes. The corresponding boundary conditions are as follows [14]:

(1) The first boundary condition is as follows: $S^{\prime}\left(t_{1}\right)=f_{1}^{\prime}$, $S^{\prime}\left(t_{n}\right)=f_{n}^{\prime}$.

(2) The second boundary condition is as follows: $S^{\prime \prime}\left(t_{1}\right)=$ $f_{1}^{\prime \prime}, S^{\prime \prime}\left(t_{n}\right)=f_{n}^{\prime \prime}$, or more special condition $S^{\prime \prime}\left(t_{1}\right)=$ $S^{\prime \prime}\left(t_{n}\right)=0$.

The spline function $S(t)$ is expressed by second derivative value of cubic spline function $S^{\prime \prime}\left(t_{j}\right)=m_{j}, j=1,2, \ldots, n$. The polynomial $S^{\prime \prime}(t)$ is less than three order on the interval $\left[t_{j}, t_{j+1}\right]$ and it is a linear polynomial in particular; more specifically,

$$
\begin{aligned}
S^{\prime \prime}(t)=m_{j} \frac{t_{j+1}-t}{h_{j}}+m_{j+1} \frac{t-t_{j}}{h_{j}} & \\
& t_{j} \leq t \leq t_{j+1}, h_{j}=t_{j+1}-t_{j} .
\end{aligned}
$$


The integration constant is acquired by integrating the above equation twice and utilizing interpolation condition. And then the cubic spline function is constructed as follows:

$$
\begin{aligned}
S(t)= & m_{j} \frac{\left(t_{j+1}-t\right)^{3}}{6 h_{j}}+m_{j+1} \frac{\left(t-t_{j}\right)^{3}}{6 h_{j}} \\
& +\left(y_{j}-\frac{m_{j} h_{j}^{2}}{6}\right) \frac{t_{j+1}-t}{h_{j}} \\
& +\left(y_{j+1}-\frac{m_{j+1} h_{j}^{2}}{6}\right) \frac{t-t_{j}}{h_{j}}
\end{aligned}
$$

where $m_{j}(j=1,2, \ldots, n)$ are unknown. Thankfully, $m_{j}$ are obtained by adopting the first and second boundary condition. As the expression of $S(t)$ is different on the interval $\left[t_{j-1}, t_{j}\right]$ and $\left[t_{j}, t_{j+1}\right]$, we also ensure its smoothness at nodes $t_{j}$, namely, the smooth condition on the interpolation nodes: $S_{j}^{\prime}\left(t_{j}-0\right)=S_{j}^{\prime}\left(t_{j}+0\right)$. The tridiagonal equations about $m_{j}$ can be obtained from the above conditions. The equations have a unique solution due to the fact that coefficient matrix of the equations is diagonally dominant matrix. We can obtain $m_{j}$ based on pursing method [15] and then put $m_{j}$ into (6); the cubic spline interpolation function $S(t)$ is acquired at last.

\section{Theoretical Results}

Theorem 3. If the integrand in (3) is replaced by the constructed cubic spline function (6), then the relationship of states at adjacent moment can be approximately represented as

$$
x_{j+1} \approx x_{j}+\frac{h_{j}^{2}}{12}\left(m_{j}+m_{j+1}\right)\left(\frac{h_{j}}{2}-1\right)+f_{j} .
$$

Proof. Now, the integrand in (3) is approximated by $S(t), t_{j}$ are interpolation nodes, and $h_{j}=t_{j+1}-t_{j}$; then

$$
\begin{aligned}
x\left(t_{j+1}\right)-x\left(t_{j}\right)=\int_{t_{j}}^{t_{j+1}} f(x(t), t) \mathrm{d} t \approx \int_{t_{j}}^{t_{j+1}} S(t) \mathrm{d} t \\
\quad=\int_{t_{j}}^{t_{j+1}}\left[m_{j} \frac{\left(t_{j+1}-t\right)^{3}}{6 h_{j}}+m_{j+1} \frac{\left(t-t_{j}\right)^{3}}{6 h_{j}}\right. \\
+\left(y_{j}-\frac{m_{j} h_{j}^{2}}{6}\right) \frac{t_{j+1}-t}{h_{j}} \\
\left.+\left(y_{j+1}-\frac{m_{j+1} h_{j}^{2}}{6}\right) \frac{t-t_{j}}{h_{j}}\right] \mathrm{d} t=\int_{t_{j}}^{t_{j+1}} m_{j} \\
\quad+\frac{\left(t_{j+1}-t\right)^{3}}{6 h_{j}} \mathrm{~d} t+\int_{t_{j}}^{t_{j+1}} m_{j+1} \frac{\left(t-t_{j}\right)^{3}}{6 h_{j}} \mathrm{~d} t+\int_{t_{j}}^{t_{j+1}}\left(y_{j}\right. \\
\left.\quad-\frac{m_{i} h_{j}^{2}}{6}\right)^{\frac{t_{j+1}}{h_{j}}} \mathrm{~d} t+\int_{t_{j}}^{t_{j+1}}\left(y_{j+1}-\frac{m_{j+1} h_{j}^{2}}{6}\right)
\end{aligned}
$$

$$
\begin{aligned}
& \cdot \frac{t-t_{j}}{h_{j}} \mathrm{~d} t=\frac{h_{j}^{3}}{24}\left(m_{j}+m_{j+1}\right)+y_{j}-\frac{h_{j}^{2}}{12}\left(m_{j}\right. \\
& \left.+m_{j+1}\right)=\frac{h_{j}^{2}}{12}\left(m_{j}+m_{j+1}\right)\left(\frac{h_{j}}{2}-1\right)+y_{j} .
\end{aligned}
$$
then

According to interpolation condition $y_{j}=f\left(x\left(t_{j}\right), t_{j}\right)$,

$$
\begin{aligned}
x\left(t_{j+1}\right)-x\left(t_{j}\right) \approx & \frac{h_{j}^{2}}{12}\left(m_{j}+m_{j+1}\right)\left(\frac{h_{j}}{2}-1\right) \\
& +f\left(x\left(t_{j}\right), t_{j}\right) .
\end{aligned}
$$

In the approximate equality equation (9), $x\left(t_{j}\right)$ is replaced by $x_{j}, f$ is represented by $f_{j}$, and then

$$
x_{j+1} \approx x_{j}+\frac{h_{j}^{2}}{12}\left(m_{j}+m_{j+1}\right)\left(\frac{h_{j}}{2}-1\right)+f_{j} .
$$

Theorem 4. If $S$ is a kind of cubic spline function as form (6), then the nonlinear dynamic system $f$ can be approximated by $S$ with the error:

$$
R(t)=S(t)-f(t) \leq \frac{1}{6} h^{4} w\left(f^{(4)}, h\right)-\frac{h^{4}}{24} f^{(4)}(t),
$$

where $0 \leq u \leq 1, t \in\left[t_{j}, t_{j+1}\right], j=1,2, \ldots, n$, and step length $h>0$.

Proof. According to (6) and Peano theorem [16], we can know that if $f \in C^{4}[0,1], t \in\left[t_{j}, t_{j+1}\right]$, then $f(x, t)$ is given by

$$
\begin{aligned}
& f(x, t)=\left(f_{j}^{\prime \prime}-\frac{h^{2}}{12} f_{j}^{(4)}\right) \frac{\left(t_{j+1}-t\right)^{3}}{6 h}+\left(f_{j+1}^{\prime \prime}-\frac{h^{2}}{12}\right. \\
& \left.\cdot f_{j+1}^{(4)}\right) \frac{\left(t-t_{j}\right)^{3}}{6 h}+\left[f_{j}-\frac{h^{2}}{6}\left(f_{j}^{\prime \prime}-\frac{h^{2}}{12} f_{j+1}^{(4)}\right)\right] \\
& \cdot \frac{\left(t_{j+1}-t\right)}{h}+\left[f_{j+1}-\frac{h^{2}}{6}\left(f_{j+1}^{\prime \prime}-\frac{h^{2}}{12} f_{j+1}^{(4)}\right)\right. \\
& \cdot \frac{\left(t-t_{j}\right)}{h} \\
& -\int_{0}^{1}\left[\left(t_{j+1}-v\right)^{3}-h^{2}\left(t_{j+1}-v\right)\right] \cdot f^{(4)}(v) \mathrm{d} v \\
& \left.\cdot \frac{\left(t-t_{j}\right)}{6 h}\right]+\frac{1}{6} \int_{0}^{1}(t-v)^{3} f^{(4)}(v) \mathrm{d} v \\
& +\frac{h}{72}\left[\left(t-t_{j}\right)^{3}-\left(t-t_{j}\right) h^{2}\right]\left(f_{j+1}^{(4)}-f_{j}^{(4)}\right)+\frac{h^{2}}{24}(t \\
& \left.-t_{j}\right)^{2} f_{j}^{(4)}-\frac{h^{3}}{24}\left(t-t_{j}\right)^{2} f_{j}^{(4)}-\int_{0}^{1}\left(t_{j+1}-v\right)
\end{aligned}
$$




$$
\begin{aligned}
& f^{(4)}(v) \mathrm{d} v \frac{\left(t-t_{j}\right)^{3}}{6 h} \\
& R(t)=S(t)-f(t)=\frac{h^{2}}{6} \widetilde{m}_{j}\left[(1-u)^{3}-(1-u)\right]+\frac{h^{2}}{6} \\
& \cdot \widetilde{m}_{j+1}\left(u^{3}-u\right)+\frac{h^{4}}{6} \int_{0}^{1}(u-v)^{3} f^{(4)}\left(t_{j}+h v\right) \mathrm{d} v \\
& +\frac{h^{4} u^{3}}{6} \int_{0}^{1}(1-v) f^{(4)}\left(t_{j}-h v\right) \mathrm{d} v+\frac{h^{4}}{6} u \\
& \cdot \int_{0}^{1}\left[(1-v)^{3}-(1-v) f^{(4)}\left(t_{j}+h v\right)\right] \mathrm{d} v \\
& -\frac{h^{4}}{72}\left(u^{3}-u\right)\left(f_{j+1}^{(4)}-f_{j}^{(4)}\right)+\frac{h^{4}}{24}\left(u-u^{2}\right) f_{j}^{(4)} \\
& =\frac{h^{2}}{6} \widetilde{m}_{j}\left[(1-u)^{3}-(1-u)\right]+\frac{h^{2}}{6} \widetilde{m}_{j+1}\left(u^{3}-u\right) \\
& +\frac{h^{4}}{6} \int_{0}^{1}\left[(u-v)^{3}-u^{3}(1-v)\right] \\
& \cdot\left[f^{(4)}\left(t_{j}+h u\right)-f^{(4)}\left(t_{j}+h v\right)\right] \mathrm{d} v+\frac{h^{4}}{6} \\
& \cdot u \int_{0}^{1}\left(v^{3}-v\right)\left[f^{(4)}\left(t_{j}-h v\right)-f^{(4)}\left(t_{j}\right)\right] \mathrm{d} v \\
& +\frac{h^{4}}{24} u^{2}\left[f^{(4)}\left(t_{j+1}\right)-f^{(4)}\left(t_{j}\right)\right]-\frac{h^{4}}{72}\left(u^{3}-u\right) \\
& \cdot\left[f_{j+1}^{(4)}\left(t_{j+1}\right)-f_{j}^{(4)}\left(t_{j}\right)\right]-\frac{h^{4}}{24} f^{(4)}(t)\left(u^{4}-2 u^{3}\right. \\
& \left.+u^{2}\right)=-\frac{h^{4}}{24} f^{(4)}(t)\left(u^{4}-2 u^{3}+u^{2}\right)+\frac{h^{4}}{24} \\
& \cdot w\left(f^{(4)}, h\right) \theta_{j}(t)\left[-u^{4}-\frac{5}{3} u^{3}-\frac{5}{3} u^{2}+4 u\right] .
\end{aligned}
$$

Due to the fact that integral form is very complicated and $\theta_{j}(t)$ does not have specific expression, $\left|\theta_{j}(x) \leq 1\right|$ can be estimated. When $0 \leq u \leq 1,0 \leq-u^{4}-5 / 3 u^{3}-5 / 3 u^{2}+4 u \leq 4$, and $0 \leq u^{4}-2 u^{3}+u^{2} \leq 1$, hence it may be

$$
\begin{aligned}
R(t)= & S(t)-f(t) \\
= & -\frac{h^{4}}{24} f^{(4)}(t)\left(u^{4}-2 u^{3}+u^{2}\right) \\
& +\frac{h^{4}}{6} w\left(f^{(4)}, h\right) \phi_{j, 0}(t),
\end{aligned}
$$

where $\phi_{j, 0}(t)=1 / 4 \theta_{j}(t)\left[-u^{4}-5 / 3 u^{3}-5 / 3 u^{2}+4 u\right]$ and $\left|\phi_{j, 0}\right| \leq$ 1 .

And finally, $R(t) \leq 1 / 6 h^{4} w\left(f^{(4)}, h\right)-1 / 24 h^{4} f^{(4)}(t)$.

Next, the second-order smoothness property of spline function [17] is given below.
Theorem 5. The cubic spline function $S(t)$ defined on interval $[a, b]$ meets interpolation condition $S\left(x_{j}\right)=y_{j}, j=1,2, \ldots, n$. Then the function $f(x) \in C^{n}[a, b]$ satisfies the above condition: $f\left(x_{j}\right)=y_{j}, j=1,2, \ldots, n$.

We have that

$$
\int_{a}^{b}\left[S^{(n)}(x)\right]^{2} \mathrm{~d} x \leq \int_{a}^{b}\left[f^{(n)}(x)\right]^{2} \mathrm{~d} x .
$$

Meanwhile, $\int_{a}^{b}\left[S^{(n)}(x)\right]^{2} \mathrm{~d} x=\int_{a}^{b}\left[f^{(n)}(x)\right]^{2} \mathrm{~d} x$ if and only if $f(x) \equiv S(x)$.

Proof. We prove Theorem 5 by proving

$$
\int_{x_{1}}^{x_{n}}\left[S^{(n)}(x)\right]^{2} \mathrm{~d} x \leq \int_{x_{1}}^{x_{n}}\left[f^{(n)}(x)\right]^{2} \mathrm{~d} x
$$

Obviously,

$$
\begin{aligned}
& \int_{x_{1}}^{x_{n}}\left[f^{(n)}(x)\right]^{2} \mathrm{~d} x \\
& =\int_{x_{1}}^{x_{n}}\left[S^{(n)}(x)\right]^{2} \mathrm{~d} x+\int_{x_{1}}^{x_{n}}\left[f^{(n)}(x)-S^{(n)}(x)\right]^{2} \mathrm{~d} x \\
& \quad+2 \int_{x_{1}}^{x_{n}} S^{(n)}(x)\left[f^{(n)}(x)-S^{(n)}(x)\right]^{2} \mathrm{~d} x .
\end{aligned}
$$

We use integral subsection integration such that

$$
\begin{aligned}
& 2 \int_{x_{1}}^{x_{n}} S^{(n)}(x)\left[f^{(n)}(x)-S^{(n)}(x)\right]^{2} \mathrm{~d} x \\
& \quad=2(-1)^{n-1} \sum_{j=1}^{n-1} \int_{x_{j}}^{x_{j+1}} S^{(2 n-1)}(x)\left[f^{\prime}(x)-S^{\prime}(x)\right] \mathrm{d} x,
\end{aligned}
$$

where $S^{(2 n-1)}(x)$ are constants on every interval $\left(x_{j}, x_{j+1}\right)$ and $f(x)-S(x)=0$ at interval endpoints $x_{j}, x_{j+1}$.

Consequently,

$$
\begin{aligned}
\int_{x_{1}}^{x_{n}}\left[f^{(n)}(x)\right]^{2} \mathrm{~d} x= & \int_{x_{1}}^{x_{n}}\left[S^{(n)}(x)\right]^{2} \mathrm{~d} x \\
& +\int_{x_{1}}^{x_{n}}\left[f^{(n)}(x)-S^{(n)}(x)\right]^{2} \mathrm{~d} x .
\end{aligned}
$$

Namely, $\int_{x_{1}}^{x_{n}}\left[S^{(n)}(x)\right]^{2} \mathrm{~d} x \leq \int_{x_{1}}^{x_{n}}\left[f^{(n)}(x)\right]^{2} \mathrm{~d} x$.

Let $n=2$ in Theorem 5; we can obtain

$$
\int_{a}^{b}\left[S^{\prime \prime}(x)\right]^{2} \mathrm{~d} x \leq \int_{a}^{b}\left[f^{\prime \prime}(x)\right]^{2} \mathrm{~d} x .
$$

The next section will cover two numerical examples, and the result reflects the advantages of cubic spline function in solving the nonlinear dynamic system. 


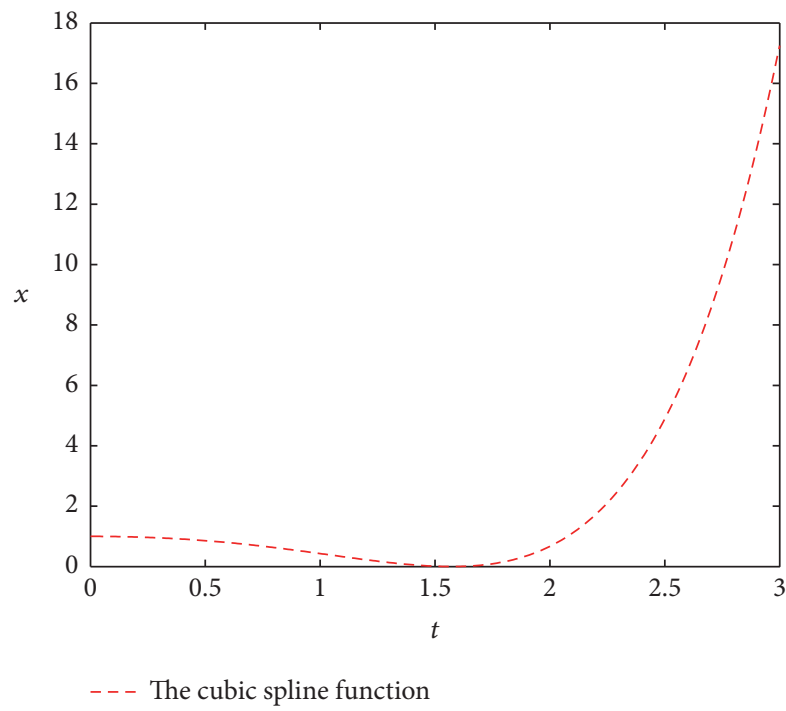

FIgURE 1: The curve of cubic spline interpolation function.

TABLE 1: Mean square errors of several numeric methods for firstorder nonlinear dynamic system.

\begin{tabular}{lc}
\hline Numeric method & Mean square error \\
\hline Fourth-order Runge-Kutta method & $1.2415 \times 10^{-4}$ \\
Trapezoid method & $9.1020 \times 10^{-3}$ \\
Euler method & $1.6205 \times 10^{-2}$ \\
Cubic spline function & $8.0240 \times 10^{-5}$ \\
\hline
\end{tabular}

\section{Numerical Results}

Example 1. Given a nonlinear dynamic system with an initial value (20), then we solve the numeric solution by using a variety of methods and compare them.

$$
\begin{aligned}
x^{\prime} & =x-e^{t} \cos t, \\
x(0) & =1,
\end{aligned}
$$

$$
0 \leq t \leq 3
$$

The numeric curve of cubic spline interpolation function is shown in Figure 1, and the approximate solution and exact solution of nonlinear dynamic system based on cubic spline interpolation function are given in Figure 2.

In the next moment, we solve numeric solution by adopting fourth-order Runge-Kutta method, trapezoidal algorithm, and Euler method and then comparing with cubic spline interpolation function. The mean square error and the curve of numeric method are given at last; specifically see Table 1 and Figure 3.

The curves of different approximation level by several numeric methods are shown in Figure 3.

Furthermore, in order to observe approximation accuracy of nonlinear dynamic system by different numeric methods intuitively, Figure 3(a) has been magnified in different proportions, and then we can obtain Figures 3(b), 3(c), 3(d), 3(e), and 3(f). We can find out Euler method

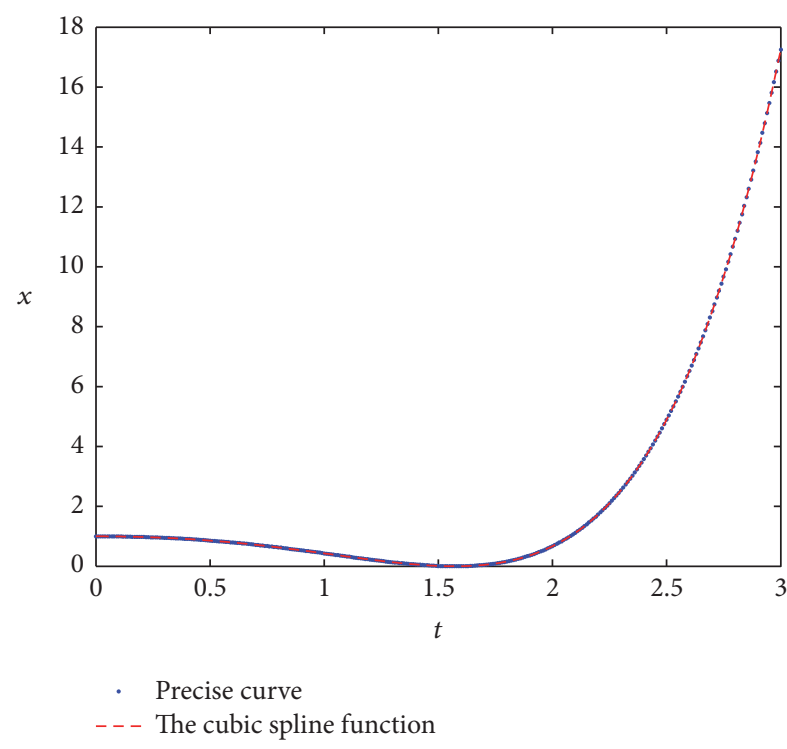

FIGURE 2: The approximate solution and precise curve.

TABLE 2: Mean square errors of several numeric methods for the second-order nonlinear dynamic system.

\begin{tabular}{lc}
\hline Numeric method & Mean square error \\
\hline Fourth-order Runge-Kutta method & 0.1662 \\
Euler method & 0.5479 \\
Adams algorithm & 0.3792 \\
Cubic spline interpolation function & 0.0179 \\
\hline
\end{tabular}

has low approximate accuracy and is great in error. The precision of the fourth-order Runge-Kutta method is also high and the calculation speed is better than trapezoidal algorithm. Although we can intuitively observe that fourthorder Runge-Kutta method has good precision compared to other methods, which is inferior to the proposed method in this paper from Figure 3.

Example 2. A second-order nonlinear dynamic system

$$
\begin{aligned}
x^{\prime \prime}+x & =2 e^{-x}(x-1), \\
x(0) & =1, \\
x^{\prime}(0) & =1,
\end{aligned}
$$

$$
0 \leq x \leq 10
$$

is considered, and we solve the numeric solution of the system. Next, several different numeric methods will be used to solve this system; mean square errors and the curves of numeric approximation will be given at last, specifically shown in Table 2 and Figure 4.

It can be seen from Figure 4 that the approximation ratio of second-order nonlinear dynamic system based on cubic spline interpolation function is better than other numeric methods, which avoids the Runge phenomenon on account of the limitation of the interpolation condition. It can approach 


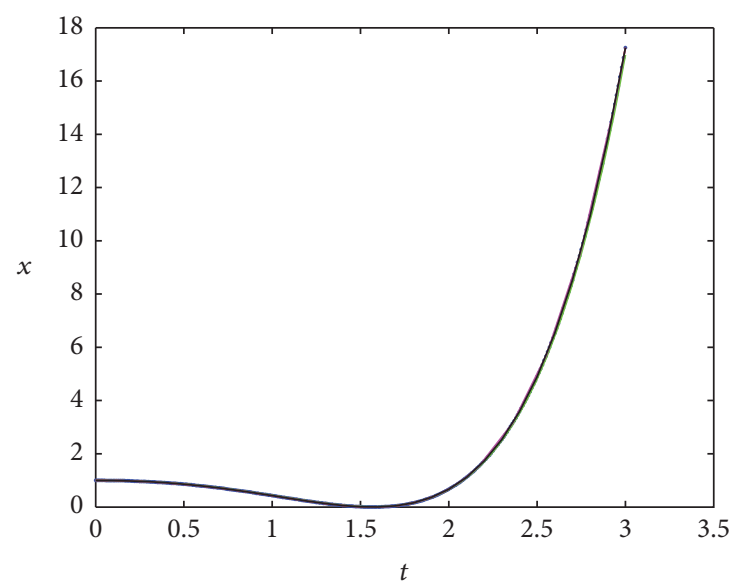

$\begin{array}{ll}\text { Precise curve } & \text { Trapezoid method } \\ --- \text { The cubic spline function } & \text { Runge-Kutta method } \\ \text { - Euler method } & \end{array}$

(a)

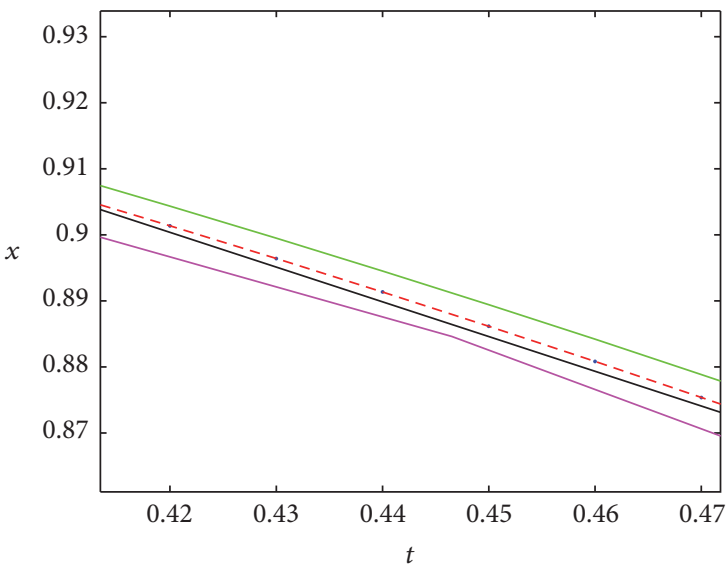

- Precise curve - Trapezoid method

- - - The cubic spline function — Runge-Kutta method _ Euler method

(c)

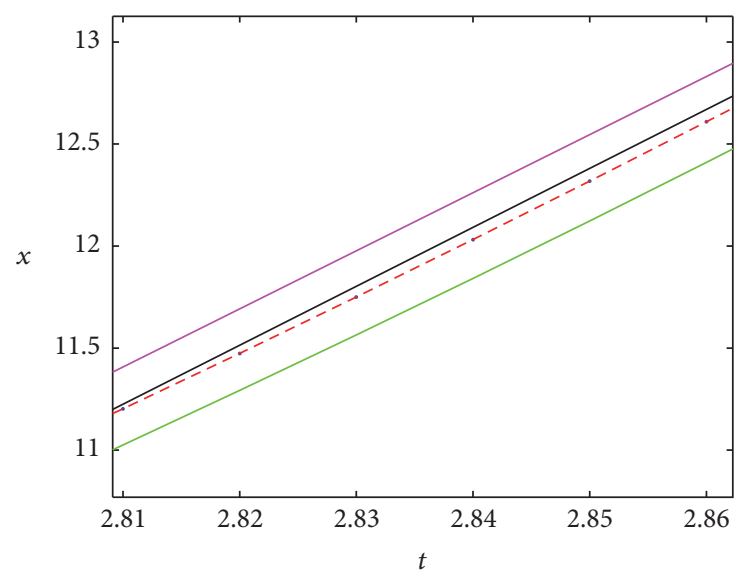

- Precise curve - Trapezoid method

- - - The cubic spline function _ Runge-Kutta method _ Euler method

(e)

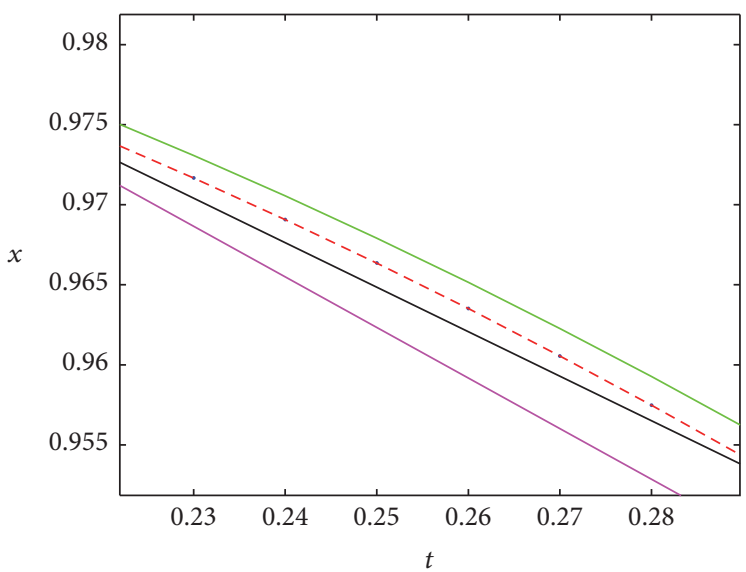

- Precise curve - Trapezoid method

- - The cubic spline function _ Runge-Kutta method _ Euler method

(b)

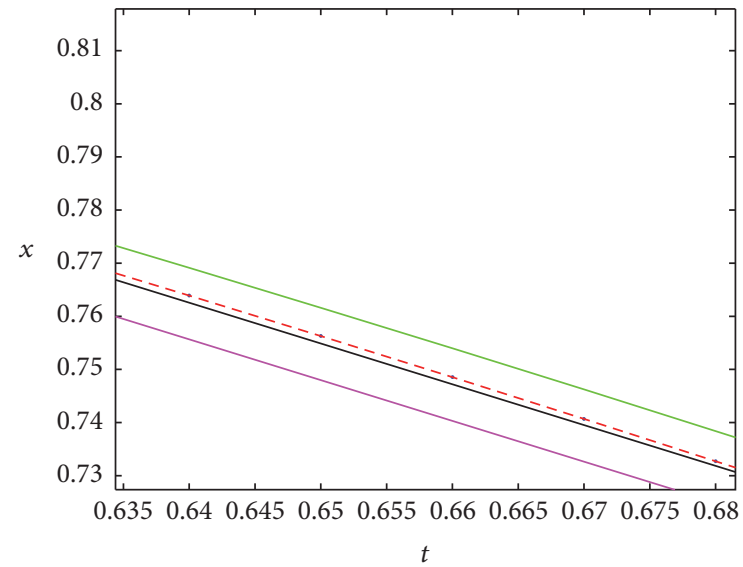
- Precise curve - Trapezoid method
- - - The cubic spline function _ Runge-Kutta method _ Euler method

(d)

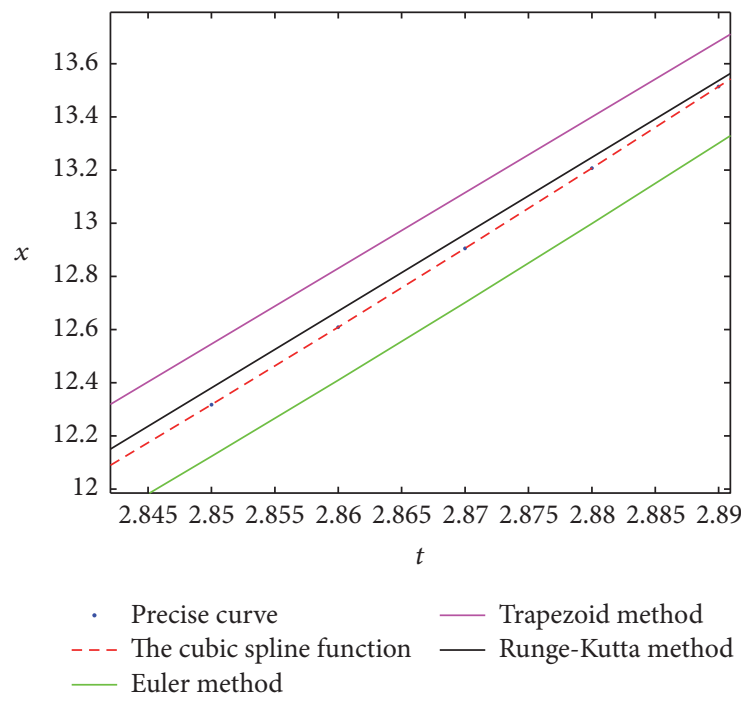

(f)

Figure 3: The different approximation level of several numeric methods. Note: (b), (c), (d), (e), and (f) are obtained from (a), which has been magnified in different proportions. 


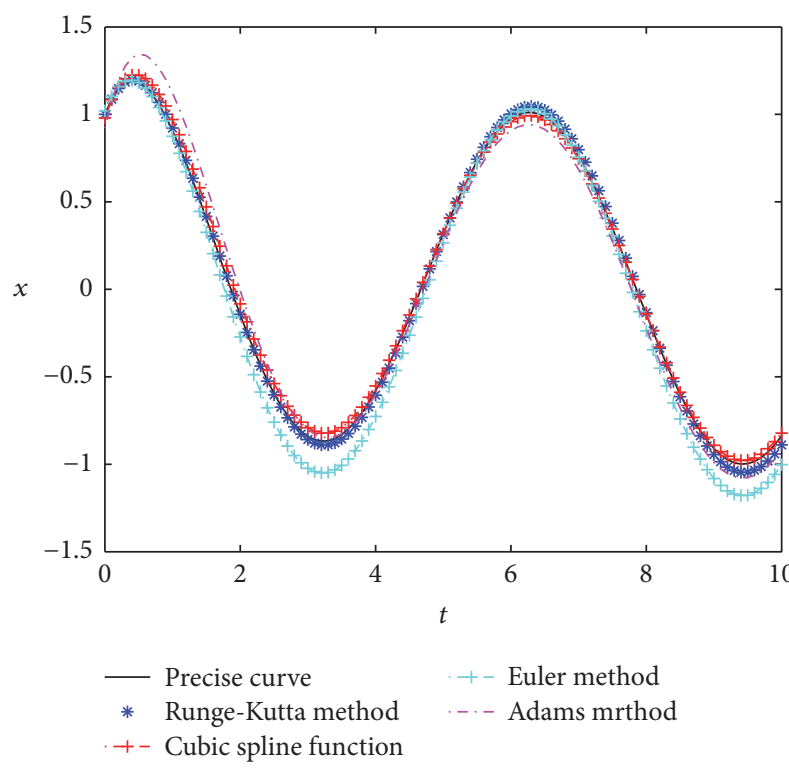

(a)

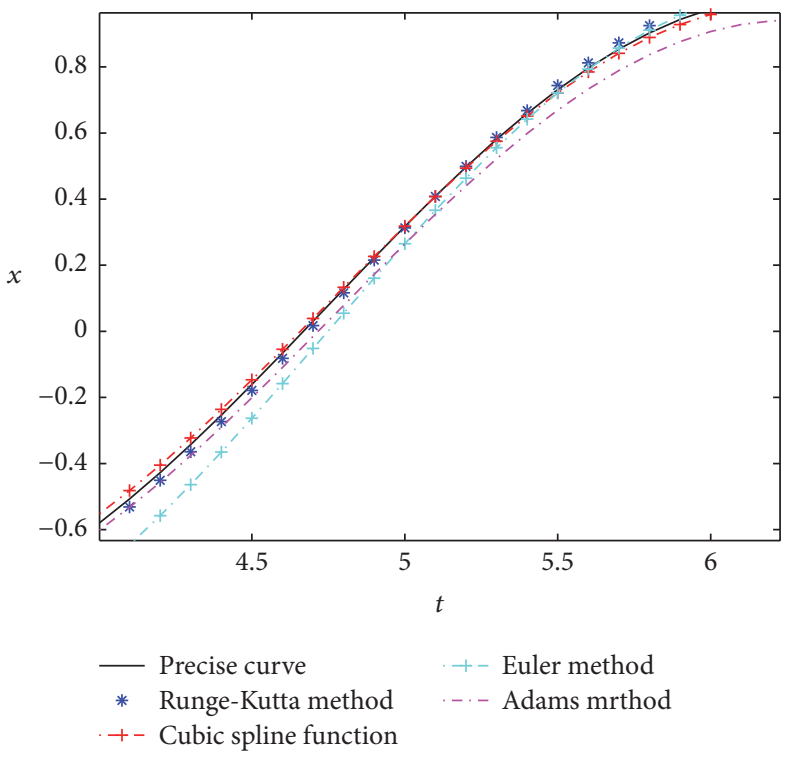

(c)

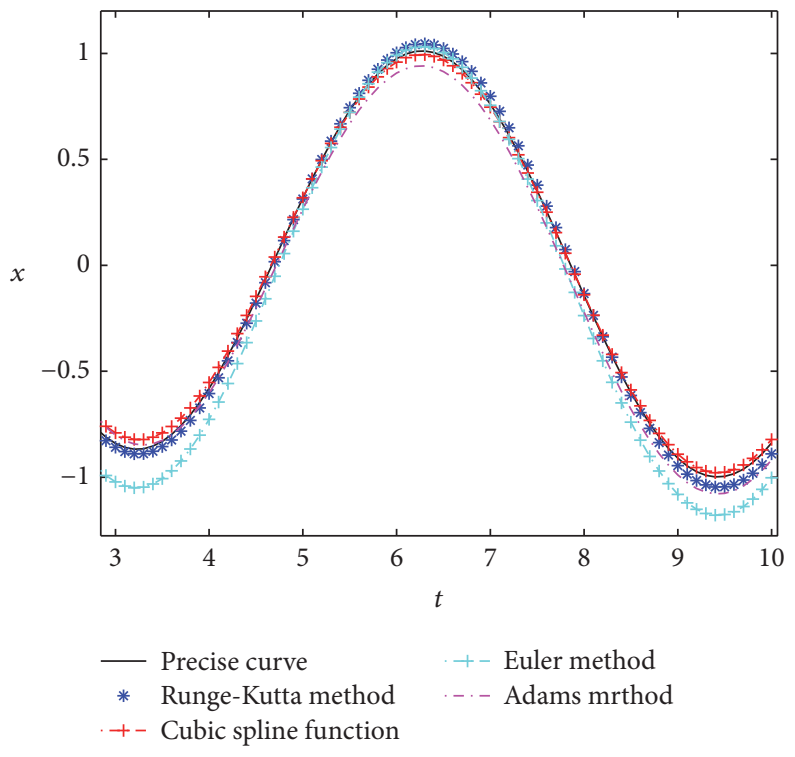

(b)

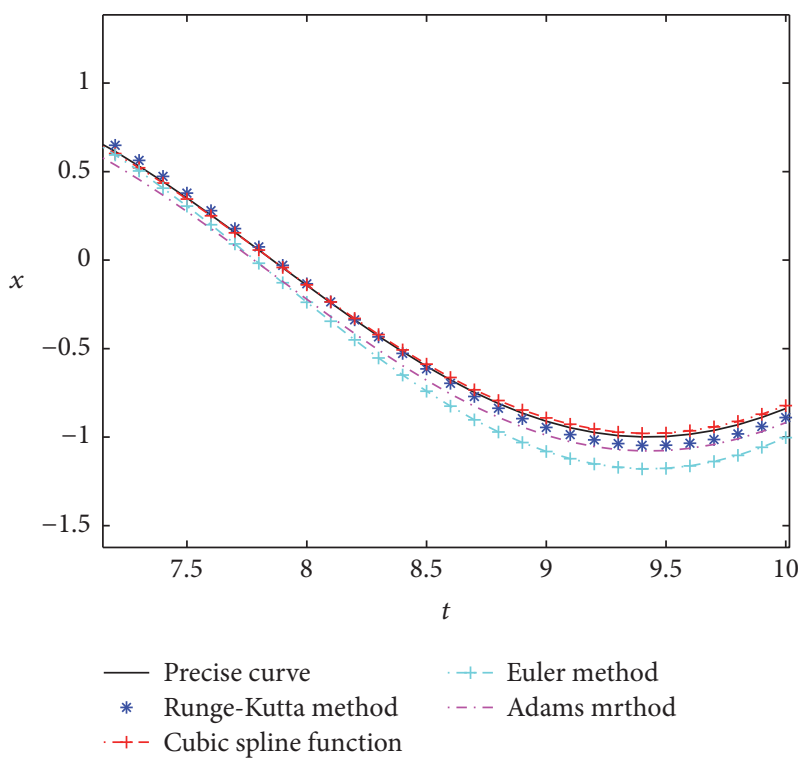

(d)

FIGURE 4: The numeric approximation curves by different methods. Note: (b), (c), and (d) are obtained from (a), which has been magnified to some extent.

the analytic solution of second-order nonlinear dynamic system with a high degree and is simple in calculation.

\section{Conclusions and Prospects}

We have discussed the numerical method based on cubic spline interpolation function to solve the numeric solution of nonlinear dynamic system in this paper. The basic theories and knowledge are introduced primarily. In the next moment, the cubic spline function is constructed according to the boundary conditions and some theory results are also given. Finally, we also demonstrate two numerical examples to reveal the effectiveness of the method proposed in this paper.
The results indicate that the proposed method based on cubic spline interpolation function is obviously advantageous compared with other methods, which has quick calculation speed and avoids the Runge phenomenon.

We finish this paper with the following prospects:

(a) We only give the upper approximation of the constructed cubic spline function in this paper. Therefore, we hope to study the lower approximation in the further study.

(b) The main theories only applied to first- and secondorder systems, but there are many of the more complex systems in actual engineering. Consequently, it is 
interesting and significant to extend the main theories in this paper to higher order nonlinear dynamic system.

\section{Competing Interests}

The authors declare that they have no competing interests.

\section{Acknowledgments}

This work was supported by the National Natural Science Foundation of China (Grant nos. 11261042 and 61662060).

\section{References}

[1] G. Chen and J. L. Moiola, "An overview of bifurcation, chaos and nonlinear dynamics in control systems," Journal of the Franklin Institute, vol. 331, no. 6, pp. 819-858, 1994.

[2] A. Farshidianfar and A. Saghafi, "Global bifurcation and chaos analysis in nonlinear vibration of spur gear systems," Nonlinear Dynamics, vol. 75, no. 4, pp. 783-806, 2014.

[3] L. V. Stepanova and S. A. Igonin, "Perturbation method for solving the nonlinear eigenvalue problem arising from fatigue crack growth problem in a damaged medium," Applied Mathematical Modelling, vol. 38, no. 14, pp. 3436-3455, 2014.

[4] J. Giné, J. Llibre, K. Wu, and X. Zhang, "Averaging methods of arbitrary order periodic solutions and integrability," Journal of Differential Equations, vol. 260, no. 5, pp. 4130-4156, 2016.

[5] H. de la Cruz, R. J. Biscay, J. C. Jimenez, and F. Carbonell, "Local linearization-Runge-Kutta methods: a class of A-stable explicit integrators for dynamical systems," Mathematical and Computer Modelling, vol. 57, no. 3-4, pp. 720-740, 2013.

[6] C. M. Duque and M. P. Almeida, "The Euler-Galerkin finite element method for a non-local coupled system of reaction diffusion type," Applied Mathematics and Computation, vol. 296, pp. 116-126, 2016.

[7] Y. H. Dai and Y. Yuan, "A nonlinear conjugate gradient method with a strong global convergence property," SIAM Journal on Optimization, vol. 10, no. 1, pp. 177-182, 1999.

[8] D. A. Mâceş and M. A. Stadtherr, "Computing fuzzy trajectories for nonlinear dynamic systems," Computers and Chemical Engineering, vol. 52, pp. 10-25, 2013.

[9] J. Guckenheimer and P. Holmes, Nonlinear Oscillations, Dynamical Systems and Bifurcations of Vector Fields, Springer, New York, NY, USA, 2013.

[10] O. Blasco, "Modulus of continuity with respect to semigroups of analytic functions and applications," Journal of Mathematical Analysis and Applications, vol. 435, no. 1, pp. 616-626, 2016.

[11] F. R. Loscalzo and T. D. Talbot, "Spline function approximations for solutions of ordinary differential equations," SIAM Journal on Numerical Analysis, vol. 4, no. 3, pp. 433-445, 1967.

[12] A. Bellour, D. Sbibih, and A. Zidna, "Two cubic spline methods for solving Fredholm integral equations," Applied Mathematics and Computation, vol. 276, no. 5, pp. 1-11, 2016.

[13] B. Sepehrian and M. K. Radpoor, "Numerical solution of nonlinear Fokker-Planck equation using finite differences method and the cubic spline functions," Applied Mathematics and Computation, vol. 262, pp. 187-190, 2015.

[14] V. Baramidze, "Smooth bivariate shape-preserving cubic spline approximation," Computer Aided Geometric Design, vol. 44, pp. 36-55, 2016.
[15] D. Wang, G. G. Wang, and G. F. Naterer, "Collaboration pursuing method for multidisciplinary design optimization problems," AIAA Journal, vol. 45, no. 5, pp. 1091-1103, 2007.

[16] P. Hájek and M. Johanis, “On Peano's theorem in Banach spaces," Journal of Differential Equations, vol. 249, no. 12, pp. 3342-3351, 2010.

[17] Z. Chungang and W. Renhong, "Functional splines with differential degree of smoothness and their applications," Computer Aided Design, vol. 40, no. 5, pp. 616-624, 2008. 


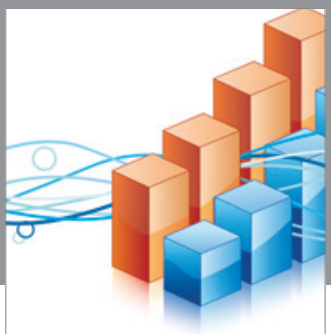

Advances in

Operations Research

vatem alat4

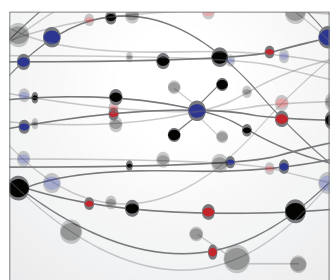

\section{The Scientific} World Journal
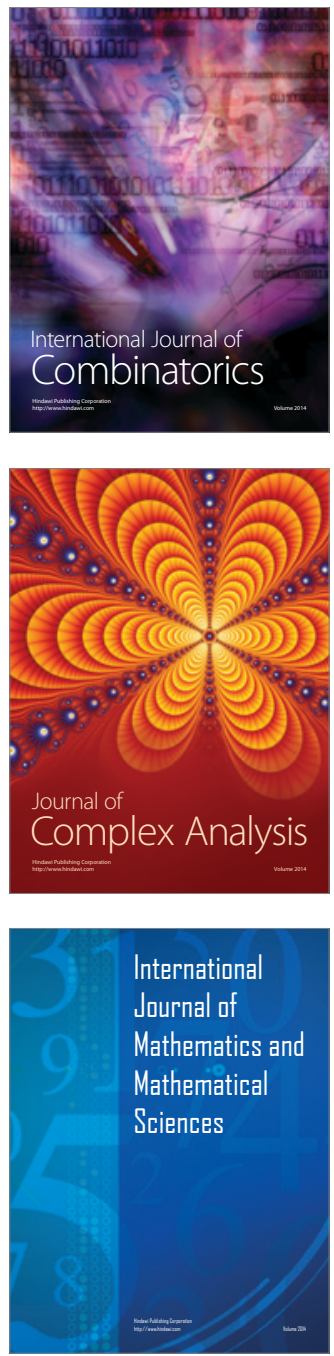
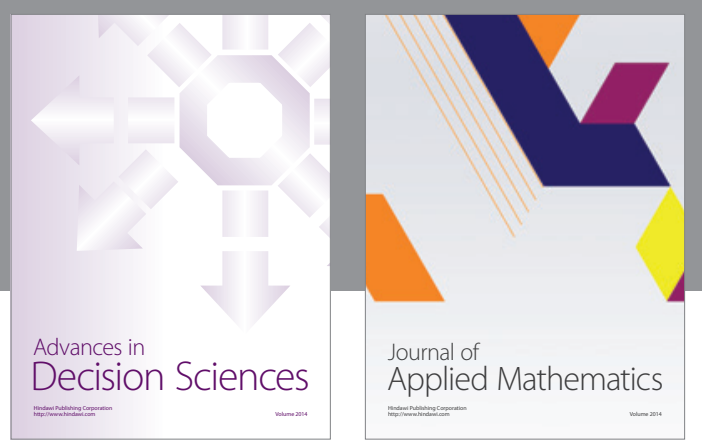

Algebra

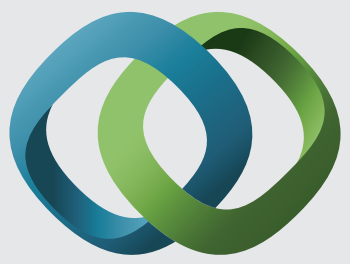

\section{Hindawi}

Submit your manuscripts at

http://www.hindawi.com
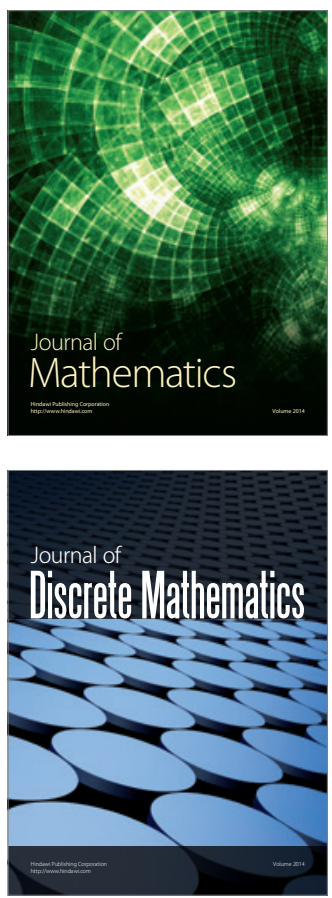

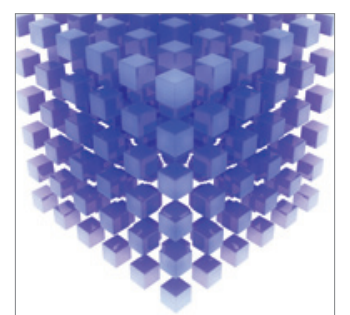

Mathematical Problems in Engineering
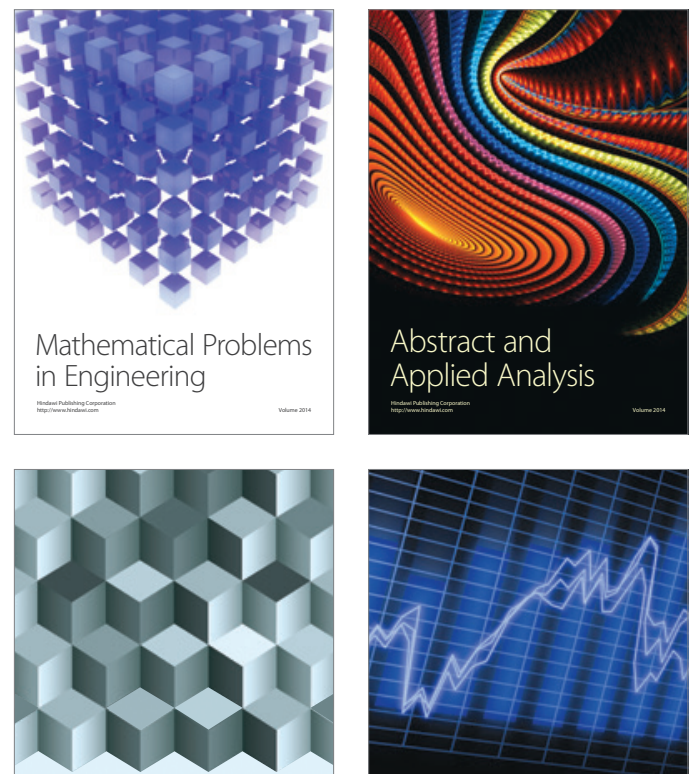

Journal of

Function Spaces

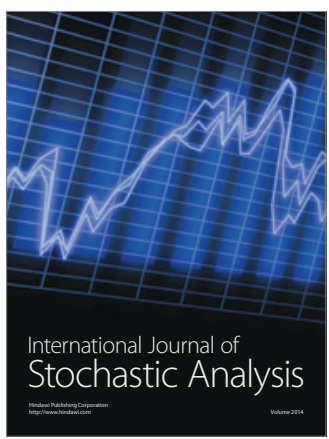

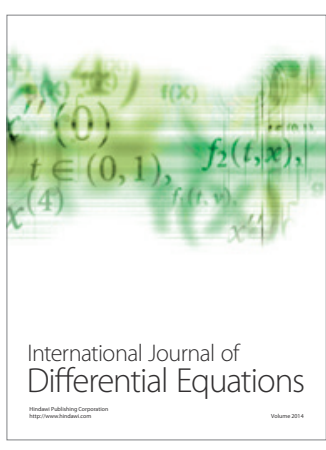
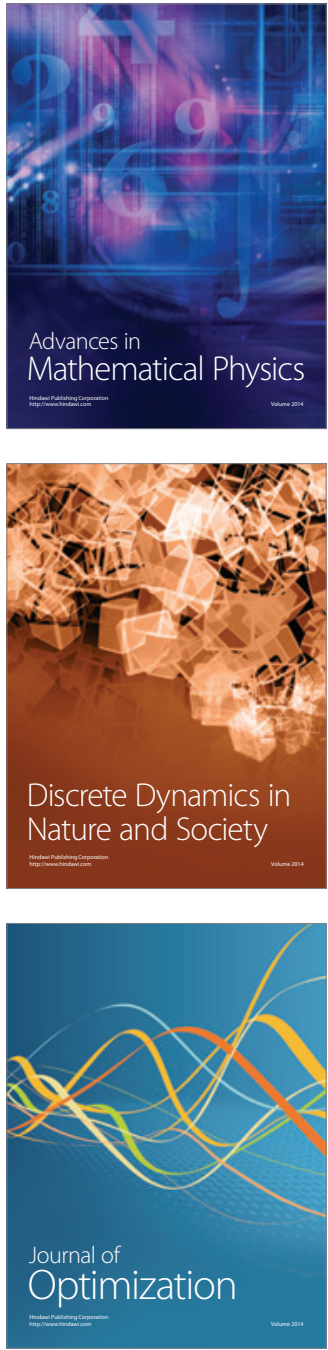